\title{
Serotypes and antimicrobial resistance patterns of avian Escherichia coli isolates from healthy and diseased chickens in Arusha city, Tanzania
}

\author{
Sanka, P.N ${ }^{1}$., Karimuribo, E.D. ${ }^{2}$ and Muhairwa, A.P. ${ }^{2}$ \\ ${ }^{1}$ Tanzania Veterinary Laboratory Agency, Zonal Office P. O. Box 1068, Arusha, Tanzania \\ ${ }^{2}$ Department of Veterinary Medicine \& Public Health, Sokoine University of Agriculture, P.O. Box 3000 Chuo \\ Kikuu, Morogoro, Tanzania
}

\begin{tabular}{|c|c|}
\hline $\begin{array}{l}\text { Article history } \\
\text { Received: } 2 \text { Aug, } 2016 \\
\text { Revised: } 22 \text { Aug, } 2016 \\
\text { Accepted: } 24 \text { Aug, } 2016\end{array}$ & 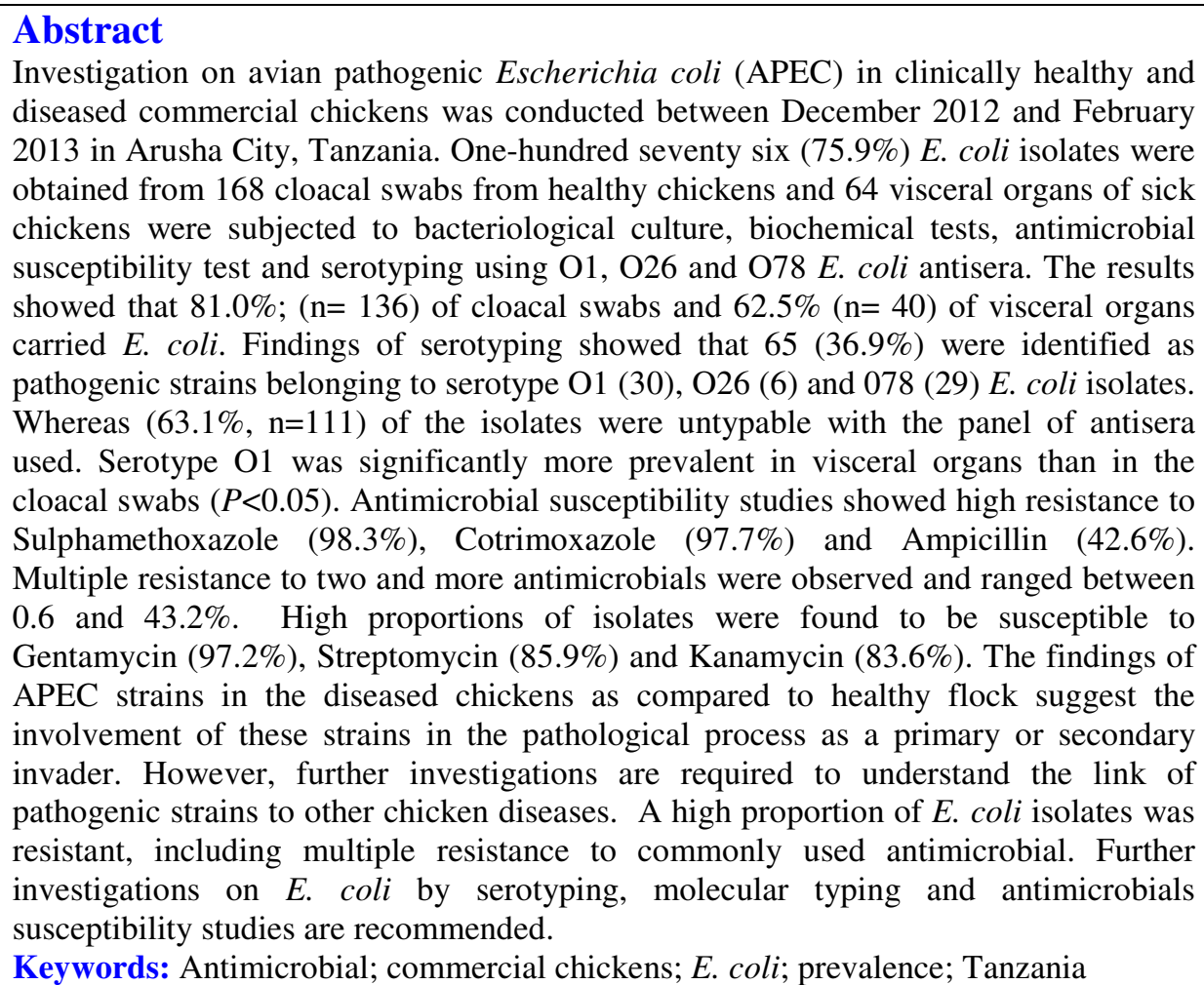 \\
\hline
\end{tabular}

To cite this article: Sanka PN, Karimuribo ED and Muhairwa AP, 2016. Serotypes and antimicrobial resistance patterns of avian Escherichia coli isolates from healthy and diseased chickens in Arusha City, Tanzania. Res. Opin. Anim. Vet. Sci., 6(6): 185-193.

*Corresponding author: Tanzania Veterinary Laboratory Agency, Zonal Office P. O. Box 1068, Arusha, Tanzania E-mail : pnsanka@yahoo.co.uk 


\section{Introduction}

Escherichia coli represent a large group of bacteria mostly residing in the gastrointestinal tracts of most mammalian species, reptiles, amphibians and birds (Fairbrother and Nadeau, 2006). E. coli are known to cause secondary bacterial infection in poultry and sometimes may constitute a primary pathogen in disease conditions including omphalitis, cellulitis, coligranuloma and colisepticemia (Gross, 1994; Shankar et al., 2010). Generally, most of the E. coli strains are considered as normal inhabitants of avian intestinal tract but some of the strains, known as avian pathogenic E. coli (APEC) can cause colibacillosis (Geidam et al., 2012). Avian colibacillosis in domestic poultry is frequently associated with strains of serotypes O78: K80, O1: K1, and O2: K1 (Khoo et al., 2010). These serotypes are highly pathogenic to poultry compared to other serotypes which are considered to be of lower pathogenicity (Allan et al., 1993; Chen et al., 2012). The majority of $E$. coli isolates are sensitive to several antimicrobial agents; however, there is an increasing number of resistance to commonly used antimicrobials (Blanco et al., 1997; Dheilly et al., 2012). In Tanzania, poultry keeping takes the form of traditional and commercial farming, which is aimed as income generation activity and source of family food. With the increase of urbanization in the country, the demand for chicken meat and other products is likely to increase. Thus, diseases including those caused by $E$. coli would affect the potential poultry industry; therefore, control of avian colibacillosis is a priority need. In Arusha city, like in other urban communities of Tanzania, keeping commercial hybrid chickens is a profitable enterprise to smallholder farmers. Poultry keepers have limited knowledge on good husbandry and the uses of appropriate drugs for treatment and control of diseases. At the moment, no information is available on the prevalence of pathogenic E. coli and antimicrobial susceptibility patterns in commercial raised chickens in Arusha City. Therefore, the present study was designed to establish the prevalence, to characterize $E$. coli isolates and to determine their antimicrobial susceptibility. The findings of this work will contribute toward advising an appropriate treatment, controlling and managing colibacillosis in the area.

\section{Materials and Methods}

\section{Study area}

This study was carried out in Arusha City, located at the base of Mount Meru, in the northern Tanzania from December, 2012 to February, 2013. The city lies between Latitude 03'16 and 03'20 South of the Equator and between Longitude 036'37 and 036'50 east of the Greenwich. The area receives bimodal rainfall, with long rainfall period commencing at the end of February until mid June, while short rains commence in October and end in December. The annual average rainfall is $800-1200 \mathrm{~mm}$ and the mean temperature ranges from $15^{\circ} \mathrm{C}$ to $30^{\circ} \mathrm{C}$. The vegetation comprises of forest, savannah grassland and woodland. Administratively, Arusha City comprises of 3 divisions (Themi, Suye and Elerai) and 17 wards namely, Sekei, Kati, Kaloleni, Ngarenaro, Themi Unga limited, Sokon 1, Daraja II, Kimandolu, Baraa Matevesi, Muriet, Terat, Burka, Sombetini and Olorien wards.

\section{Poultry production system}

The poultry keepers in Arusha city practice different type of poultry production systems. These include commercial production (broilers and layers) and extensive backyard traditional system of indigenous chickens. Both systems are constrained by the inadequacies of capacity to control diseases, poor nutrition, husbandry management and misuse of drugs.

\section{Study farm selection}

A multi-stage sampling technique was employed to obtain apparently healthy chickens for the study. Five wards based on the numerical high number of chickens possessed were purposely selected for the study. Selected wards were Kaloleni, Sekei, Themi, Unga limited and Kimandolu. The study flocks/households were established by listing all households with broilers and layers in the 5 selected wards using ward extension officers, whereby a total of 48 households were identified of which 25 households were keeping broilers and 23 households keeping layers. The average number of broiler chickens was 325 and layers was 360 per household. Lastly, 6 households (3 broilers and 3 layers) in each ward were randomly selected using Microsoft Excel software. Overall, 30 households (15 broilers and 15 layers) were enrolled and participated in the study.

\section{Study design and sample size}

A cross-sectional study design was adopted and birds selected for the study were examined and sampled once. Sample size estimation was based on a sampling frame of 15 broilers and 15 layers farms distributed across the five selected wards using the formula $n=Z^{2}$ $\mathrm{P}$ (1-P)/ $\mathrm{d}^{2}$ developed by Pfeiffer (1999). Since the prevalence of APEC in the region was not known, the prevalence of $50 \%$ was adopted within $7 \%$ precision of the true prevalence at $95 \%$ confidence. Therefore, the calculated sample size was $1.96^{2} \times 0.5 \times 0.5 / 0.07^{2}=$ 168. However, the practical working sample was raised to 168. Additional 64 chicken carcasses submitted for disease diagnosis during the study period were also 
investigated in order to establish if pathogenic E. coli plays a role in morbidity and mortality of chickens in Arusha City.

\section{Sample and data collection}

The sample collection and information related to bird bio-data were performed by Paul N. Sanka (main author) and technical assistants (laboratory technician and field officers) were provided from zonal VIC, Arusha and City livestock office, Arusha respectively.

Cloacal swabs were aseptically collected from apparently healthy chickens of different age groups using sterile cotton wool swabs and were placed in a sterile test tube containing sterile normal saline and stored on ice in a cool box. Collected samples were transported to VIC and processed on the same day of collection. Information on antimicrobial agents commonly used for treatment during previous days/weeks was also recorded. Furthermore, postmortem examination of dead euthanized or sick chickens submitted to VIC by the poultry keepers from Arusha city based wards was also carried out. However, the information collected from the dead birds included chicken type (broilers/layers), age, ward, antibiotics used and owner. Pathological findings of each chicken carcass were recorded. After post-mortem examination, visceral organs as illustrated in Fig. 1 like liver, lung, spleen, air sacs and yolk were aseptically collected and placed in sterile petri dishes before being cultured for $E$. coli isolation on the same day.

\section{Primary isolation of $E$. coli}

Primary isolations were performed using MacConkey agar (Hi Media ${ }^{\circledR}$, Mumbai, India) and Eosin methylene blue agar (Hi Media ${ }^{\circledR}$, Mumbai, India). Cloacal swabs from healthy chickens were aseptically inoculated on both media simultaneously and incubated at $37^{\circ} \mathrm{C}$ for $18-24$ hours. For the case of visceral organs, the surface of the organ was briefly sterilized by flaming to remove normal flora. The material to be inoculated was taken from inside of the organ using a sterile wire loop, then streaked on both media simultaneously and incubated aerobically at $37^{\circ} \mathrm{C}$ for 18-24 hours, for isolation of E. coli as per standard bacteriological method described by Longman (1999).

\section{Identification of $E$. coli isolates}

Identification of $E$. coli isolates was conducted using a panel of four biochemical tests, namely, Indole, Methyl red, Voges-proskauer and Simmons citrate agar (IMVC) as per standard protocol described by Cheesbrough (2000). The presumed E. coli colonies were aseptically inoculated into the aforementioned tests media and incubated at $37^{\circ} \mathrm{C}$ for $18-24$ hours. The standard E. coli reference strains used was ATCC $25922 ®$ (Manassas, USA). Pure colonies were used for
Gram's staining for microscopic examination of the morphological characteristics as per protocol described by Merchant and Park (1967).

\section{Serotyping}

Antigen suspensions were prepared by taking four matched colonies of $E$. coli from the plate using sterile wire loop and placed in a sterile tube containing $3 \mathrm{ml}$ of sterile $0.85 \%$ normal saline. The suspensions were autoclaved at $121^{\circ} \mathrm{C}$ for 15 minutes and centrifuged at $900 \mathrm{rpm}$ for 20 minutes. The supernatants were removed aseptically using a sterile pipette and $0.5 \mathrm{ml}$ of sterile $0.85 \%$ normal saline were added to re-suspend the precipitate and mixed thoroughly. In the present study, $E$. coli isolates were serotyped using the panel of three monospecific "O" antisera of $E$. coli belonging to APEC group (O1, O26 and O78). The serotyping was conducted by standard slide agglutination test with specific antisera against the somatic antigen (Kok et al., 1996). Briefly, a drop of each antigen suspension, control organism and normal saline were placed separately onto a cleaned microscope slide followed by adding a drop of antisera on the drop of antigen suspension, control organism and normal saline. The drops on the slide were thoroughly mixed using a wooden applicator and the slide was rocked for approximately one minute. An agglutination reaction observed was considered as positive for pathogenic strains while absence of agglutination reaction was considered as negative for pathogenic strains.

\section{Antimicrobial susceptibility testing}

Antimicrobial agents commonly used by poultry keepers were used for susceptibility testing in the study. Eight panels of antimicrobial agents included in the test were Ampicillin $(25 \mu \mathrm{g})$, Tetracycline $(25 \mu \mathrm{g})$, Cotrimoxazole $\quad(25 \mu \mathrm{g}), \quad$ Streptomycin $(10 \mu \mathrm{g})$, (Kanamycin $\quad(30 \mu \mathrm{g}), \quad$ Gentamycin $\quad(10 \mu \mathrm{g})$, Sulphamethoxazole $(200 \mu \mathrm{g})$ and Chloramphenicol $(30 \mu \mathrm{g})$. Antimicrobial susceptibility discs were obtained from Hi Media ${ }^{\circledR}$ Mumbai, India. Antimicrobial susceptibility test of the E. coli isolates was done using the standard disc diffusion test as recommended by the Clinical Laboratory Standard Institute (CLSI, 2011).

Suspensions for inoculation were prepared by taking three isolated colonies from the plate using sterile wire loop and inoculated into $5 \mathrm{ml}$ normal saline followed by incubation at $37^{\circ} \mathrm{C}$ for 3 hours as described by Stephen et al. (2002). The suspension (inoculum) was thoroughly mixed by vortexing and compared with 0.5 Macfarland standard reagents. The suspension was taken from the tube using a sterile cotton swab and inoculated on Mueller Hinton agar $\left(\mathrm{Oxoid}^{\circledR}\right.$, Hampshire, UK) by streaking the entire surface of the plate (Stephen et al., 2002). The antimicrobial discs 
were applied onto the surface of the inoculated Mueller Hinton agar plates using sterile forceps and pressed down each disc to ensure complete contact with the agar surface and were incubated aerobically at $37^{\circ} \mathrm{C}$ for 18-24 hours. The diameter of the zones of complete inhibition of each disc was measured using a ruler that was placed at the back of the Petri dish in reflecting light while the discs with no zones of inhibition were also observed by eyes. The zone of inhibition around each disc was recorded and interpreted as either susceptible (S), intermediate (I) or resistant (R) based on the breakpoint of each antimicrobial agent as indicated in the guidelines of CLSI (2011).

\section{Statistical analysis}

Data were analyzed using Epi Info ${ }^{\mathrm{TM}}$ Version 7.0.8.0 statistical software (CDC, 2012). Descriptive statistics were generated where the prevalence was determined as the percentage of occurrence of an event in the sampled populations. Chi square test was used to assess for the statistical significance of the prevalence of pathogenic $E$. coli and antimicrobial susceptibility test. The level of significance was set at $\mathrm{P}<0.05$. Microsoft excel $^{\circledR}$ was used as a graphical software to produce different graphs.

\section{Results}

\section{Post-mortem findings}

In the present study, a total of 64 (32 broilers and 32 layers) chicken carcasses of different ages from 1 week to 120 weeks originating from 17 wards were examined for pathological lesions. PM findings revealed a variable gross lesion in various visceral organs. The prominent lesions observed were gross pulmonary congested, petechial haemorrhages on the spleen, congested liver, enlarged liver with necrotic foci, distended gallbladder with bile, pericarditis, eggbound peritonitis, mucoid enteritis, intestinal enteritis, fibrinous exudates on liver and unabsorbed yolk sac with yellow fluid as illustrated in (Fig. 1). Isolation rates of $E$. coli from different categories of samples

A total of $176(75.9 \%)$ E. coli isolates were obtained from 232 analysed samples (Table 1). Of the total samples, 168 were cloacal swabs collected from healthy chickens and 64 were visceral organs collected from sick chickens submitted for disease diagnosis. The $E$. coli isolation rates were categorized based on the chicken types, healthy status, locations and age group (Table 1). The E. coli isolates were recovered from broilers $(77.4 \%)$ and layers $(74.4 \%)$ and from cloacal swabs $(81.0 \%)$ of healthy chickens and visceral organs $(62.5 \%)$ of sick chickens (Table 1). Bird type (broiler or layer) and administrative location were not significantly associated with isolation rate of $E$. coli in this study (P>0.05) (Table 1).

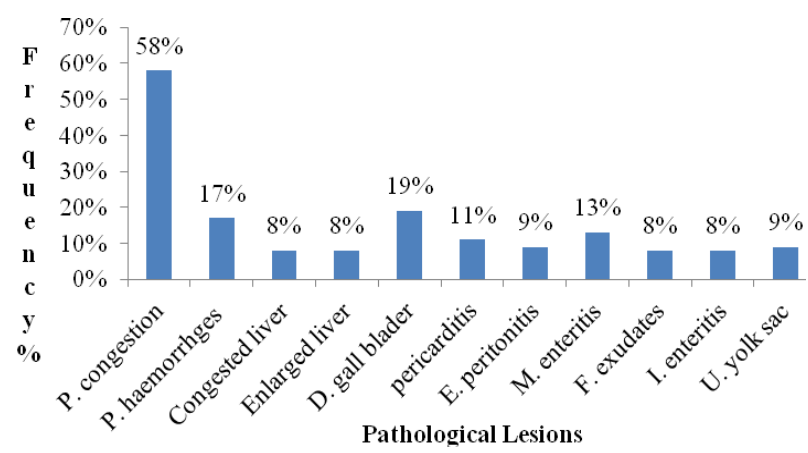

Fig. 1: Detected post-mortem lesions and their distribution

Table 1: Isolation rates of $E$. coli from different categories of chickens

\begin{tabular}{lllll}
\hline & & $\begin{array}{l}\text { No. of } \\
\text { samples } \\
\text { tested }\end{array}$ & $\begin{array}{l}\text { No. of } \\
\text { E. coli } \\
\text { Isolates (\%) }\end{array}$ & P-value \\
\hline Parameter & Level & 115 & $89(77.4)$ & $0.5902^{\mathrm{ns}}$ \\
\hline Chicken type broilers & 117 & $87(74.4)$ & \\
$(\mathrm{N}=232)$ & layers & 168 & $136(81.0)$ & \\
Sample type & cloacal swabs & $168.0033^{*}$ \\
$(\mathrm{~N}=232)$ & visceral organs & 64 & $40(62.5)$ & 0.003 \\
ward & Kaloleni & 33 & $30(90.9)$ & \\
$(\mathrm{n}=168)$ & Themi & 37 & $27(73.0)$ & \\
& Sekei & 32 & $25(78.1)$ & $0.1436^{\mathrm{ns}}$ \\
& Unga limited & 33 & $30(90.9)$ & \\
& Kimandolu & 33 & $29(87.9)$ & \\
\hline
\end{tabular}

Key: $*$ = statistically significant difference, ${ }^{\mathbf{n s}}=$ statistically no significant difference

Subsequently, the chickens of different ages were analysed for the occurrence of $E$. coli isolates in order to ascertain the effect of age on isolation of $E$. coli. For convenience, chickens with an age ranging from 1 week to 120 weeks were combined in 5 groups and compared for statistical significance. A similar trend of E. coli isolates were observed in different age groups, of which there were no statistically significant differences observed between different age groups in respect to $E$. coli isolates ( $\mathrm{P}>0.05)$, (Fig. 2).

\section{Isolates serotypes}

In the present study, out of $176 \mathrm{E}$. coli isolates serotyped, $65(36.9 \%)$ belonged to $\mathrm{O} 1, \mathrm{O} 26$ or $\mathrm{O} 78$ serotype. The distribution of the isolates was 30 $(17.0 \%)$ to serotype $01,29(16.5 \%)$ to serotype 078 and $6(3.4 \%)$ to serotype $\mathrm{O} 26$ (Table 2 ). The remaining $111(63.1 \%)$ E. coli isolates were un-typable with the set of antisera used in this study. The isolation rate of serotype $\mathrm{O} 1$ was significantly higher $(\mathrm{P}<0.05)$ in visceral organs than in cloacal swabs while for the serotype $\mathrm{O} 26$ and $\mathrm{O} 78$ there was no significant difference in the isolation rate between cloacal swabs and visceral organs $(\mathrm{P}>0.05)$. Overall, the isolation rates of serotypes were significantly higher $(\mathrm{P}<0.05)$ in the visceral organs than in the cloacal swabs (Table 2). 


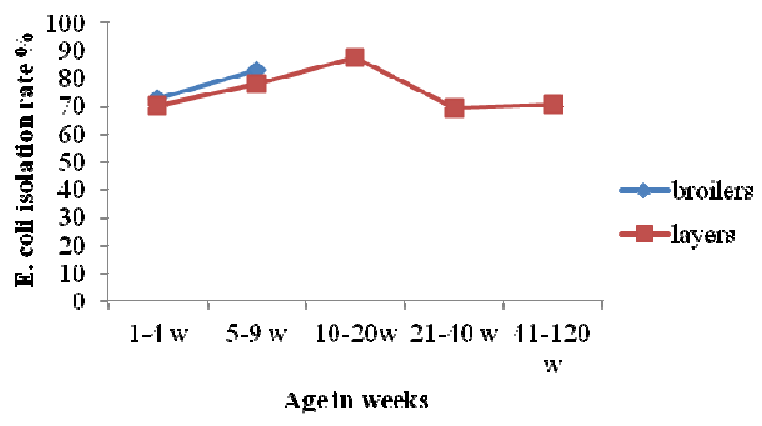

Fig. 2: Effect of age on isolation of $E$. coli

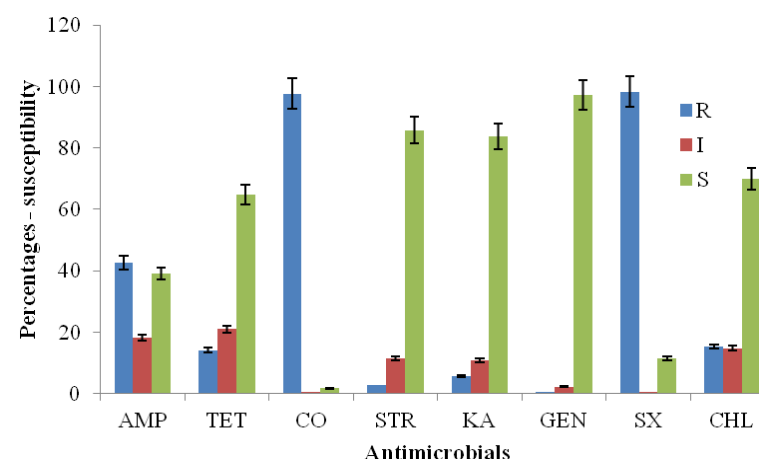

Fig. 3: Antimicrobial susceptibility patterns of E. coli isolates from chickens

Key: $\mathrm{AMP}=$ ampicillin $(25 \mu \mathrm{g}), \mathrm{TET}=$ tetracycline $(25 \mu \mathrm{g})$, $\mathrm{CO}=$ cotrimoxazole $(25 \mu \mathrm{g}), \mathrm{KA}=$ kanamycin $(30 \mu \mathrm{g}), \mathrm{STR}=$ streptomycin $(10 \mu \mathrm{g})$, GEN = gentamycin $(10 \mu \mathrm{g}), \mathrm{SX}=$ sulphamethoxazole $(200 \mu \mathrm{g})$ and $\mathrm{CHL}=$ chloramphenicol $(30 \mu \mathrm{g}) . \mathrm{S}=$ susceptible, $\mathrm{R}=$ resistant and $\mathrm{I}=$ intermediate.

Table 2: Serotypes obtained from cloacal swabs and visceral organs

\begin{tabular}{lcccr}
\hline $\begin{array}{l}\text { Sero- } \\
\text { types }\end{array}$ & $\begin{array}{c}\text { Overall } \\
(\mathrm{n}=176)(\%)\end{array}$ & $\begin{array}{c}\text { Cloacal swabs } \\
(\mathrm{n}=136)(\%)\end{array}$ & $\begin{array}{c}\text { Visceral } \\
\text { organs } \\
(\mathrm{n}=40)(\%)\end{array}$ & P-value \\
\hline O1 & $30(17.0)$ & $19(14.0)$ & $11(27.5)$ & $0.0461^{*}$ \\
O26 & $6(3.4)$ & $5(3.7)$ & $1(2.5)$ & $0.7193^{\mathrm{ns}}$ \\
O78 & $29(16.5)$ & $20(14.7)$ & $9(22.5)$ & $0.2241^{\mathrm{ns}}$ \\
Total & $65(36.9)$ & $44(32.4)$ & $21(52.5)$ & $0.0207^{*}$ \\
\hline Key: $*$ & significant difference, ns = no significant difference
\end{tabular}

\section{Antimicrobial susceptibility results}

A total of $176 \mathrm{E}$. coli isolates were tested and the isolates were interpreted as susceptible, intermediate and resistant to each antimicrobial agent (Fig.3) depending on the recorded inhibition zone. Overall, the proportion of the highest resistance were to Sulphamethoxazole (98.3\%) followed by Cotrimoxazole (97.7\%) and Ampicillin (42.6\%). The lowest level of resistance were to Chloramphenicol $(15.3 \%)$ and Tetracycline (14.2\%) (Fig. 2). Intermediate resistance was to Tetracycline $(21 \%)$, Chloramphenicol (18.2\%) and ampicillin (14.8\%). On the other hand, highest susceptibility were to Gentamycin (97.2\%), Streptomycin (85.8\%) and Kanamycin (83.6\%) (Fig. 3).

\section{Multiple antibiotics resistant strain categorization}

Multiple antibiotic resistance patterns were categorized based on the frequency of at least two or more antimicrobials in both broilers and layers isolate (Table 3). In the present investigation, $76 \mathrm{E}$. coli isolates were resistant to 2 antibiotics followed by 54 isolates to 3 antibiotics, 28 isolates to 4 antibiotics, 3 isolates to 5 antibiotics, 1 isolate to 6 antibiotics and 1 isolate to 7 antibiotics (Table 3). The recovered multiple resistance to a combination of 4 antibiotics, namely, AMP-CO- SX-CH, in broilers isolates (11.2\%) statistically differ $(\mathrm{P}<0.05)$ than layers $(3.4 \%)$. However, there was no statistically significant difference $(\mathrm{P}>0.05)$ between broilers and layers isolates to the rest of the antimicrobial agents (Table 3 ).

\section{Antimicrobials commonly used in poultry farms during the study}

During sample collection, the information on antimicrobials commonly used in poultry farms were collected from 37 poultry keepers while no information was found from 27 out of total 63 (34 from healthy and 30 from sick flocks) poultry keepers. Antibiotics reported to be commonly used were Tetracycline (56.8\%, 21/37), Cotrimoxazole (35.1\%, 13/37), Sulphamethoxazole $(21.6 \%, 8 / 37)$, Flumequin $(13.5 \%$, $5 / 37)$ and Gentamycin $(8.1 \%, 3 / 37)$. These antibiotics were found in the stores of the poultry farms.

\section{Discussion}

The results of this study show the presence of $E$. coli isolates in cloacal samples from clinically healthy chickens and from visceral organs of sick chickens submitted for diagnosis. These findings further confirm the presence of $E$. coli in the gastrointestinal tract of healthy chickens as commensals and in visceral organs of infected birds as extra intestinal E. coli infection. The finding of the pathogenic serotypes of $E$. coli in the intestinal tract of healthy chickens and in the visceral organs of the diseased chicken implies the invasive power of the E. coli isolates. This is suggestive of involvement of these strains as either primary pathogen or secondary invader. However, more studies on the invasion pathogenic markers are needed to confirm this. In this study, the isolations of E. coli from chickens of different age groups were also comparable. The isolates showed a similar trend in all different ages, suggesting that $E$. coli infection may affect chickens of all ages as previously reported by others (Johnson et al., 2001; Ivanov, 2007).

Avian pathogenic E. coli isolates obtained in this study belonged to serotypes O1 (17.0\%), O78 (16.5\%) and $\mathrm{O} 26(3.4 \%)$. The isolation of these serotypes has been associated with disease such as colibacillosis in 
Res. Opin. Anim. Vet. Sci., 2016, 6(6): 185-193.

Table 3: Multiple antimicrobial resistance patterns

\begin{tabular}{lcccc}
\hline Antimicrobial agent - combination & Overall & Broilers & Layers & \multirow{2}{*}{ p-value } \\
\cline { 2 - 4 } & $(\mathrm{n}=176)(\%)$ & $(\mathrm{n}=89)(\%)$ & $(\mathrm{n}=87)(\%)$ & \\
\hline CO-SX & $76(43.2)$ & $38(42.7)$ & $38(43.7)$ & $0.8957^{\mathrm{ns}}$ \\
AMP-CO-SX & $37(21)$ & $17(19.1)$ & $20(23.0)$ & $0.5280^{\mathrm{ns}}$ \\
AMP-CO-SX-CH & $13(7.4)$ & $10(11.2)$ & $3(3.4)$ & $0.0489^{*}$ \\
AMP-TET-CO- SX & $11(6.3)$ & $6(6.7)$ & $5(5.7)$ & $0.7858^{\mathrm{ns}}$ \\
TET-CO-SX & $9(5.1)$ & $4(4.5)$ & $5(5.7)$ & $0.7681^{\mathrm{ns}}$ \\
CO-SX-CH & $5(2.3)$ & $1(1.1)$ & $4(4.6)$ & $0.1667^{\mathrm{ns}}$ \\
CO-STRP-SX & $3(1.7)$ & $0(0.0)$ & $3(3.4)$ & $0.0781^{\mathrm{ns}}$ \\
AMP-CO-KA-SX & $3(1.7)$ & $2(2.2)$ & $1(1.1)$ & $0.5749^{\mathrm{ns}}$ \\
TET-CO-SX-CHL & $1(0.6)$ & $0(0.0)$ & $1(1.1)$ & $0.3118^{\mathrm{ns}}$ \\
AMP-TE-CO-KA-SX & $1(0.6)$ & $0(0.0)$ & $1(1.1)$ & $0.3118^{\mathrm{ns}}$ \\
AMP-CO-STR-SX-CH & $1(0.6)$ & $1(0.07)$ & $0(0.0)$ & $0.3228^{\mathrm{ns}}$ \\
AMP-CO-GEN-SX-CH & $1(0.6)$ & $1(0.07)$ & $0(0.0)$ & $0.3228^{\mathrm{ns}}$ \\
AMP-TET-CO-KA-SX-CH & $1(0.6)$ & $1(0.07)$ & $0(0.0)$ & $0.3228^{\mathrm{ns}}$ \\
AMP-TET-CO-STR-KA-SX-CH & $1(0.6)$ & $1(0.07)$ & $0(0.0)$ & $0.3228^{\mathrm{ns}}$ \\
\hline
\end{tabular}

Key $*$ = statistically significant difference, $\mathrm{ns}=$ no statistically significant difference. AMP = ampicillin, TET = tetracycline, CO $=$ cotrimoxazole, $\mathrm{STR}=$ streptomycin, $\mathrm{KA}=$ kanamycin, $\mathrm{GEN}=$ gentamycin, $\mathrm{SX}=$ sulphamethoxazole and $\mathrm{CHL}=$ chloramphenicol.

chickens (Blanco et al., 1997; Yousseff et al., 2008; Chen et al., 2012). In the present investigation, serotypes O1, O26 and O78 were isolated from both healthy and sick chickens, but to a lower rate compared to what was reported by Yousseff et al. (2008). The observed differences could be due to the limited number of serovars panel used and partly due to differences in sampling methodology and laboratory analysis procedure which in the present study involved cloacal swabs and a little number of sick chickens while in the study by Yousseff et al. (2008) analyses a large number of visceral organs of sick chickens. The findings underline the role of E. coli from gastrointestinal tract as opportunistic and potentially systemic pathogenic in chickens. Investigation of other important pathogenic $E$. coli serovars is warranted to give more insights about these pathogens in chickens in Tanzania. The present study observed the proportion of $63.1 \%$ E. coli isolates as untypable based on the $\mathrm{O} 1, \mathrm{O} 26$ and $\mathrm{O} 78$ antisera used. Studies by D'Incau et al. (2006) and Geidam et al. (2012) showed the proportions of untypable strains to range between $49.2 \%$ and $68 \%$ in chickens similar to the findings of the present study. However, with only three serotypes investigation, caution must be applied, when compared with other studies as it reflects that a large number of $E$. coli strains are untypable. Therefore, further studies, including a wide range of serotypes to reduce the number of untypable serotypes are recommended. Broadly speaking, the recovery of $E$. coli serotypes including untypable serotypes from clinically healthy chickens in this study comprises of pathogenic and non-pathogenic E. coli strains. Thus, residing of these microorganisms in the body of healthy chickens may reflect the possibility of these serotypes to act as opportunistic pathogens to cause E. coli infection in immuno-compromised chicken as reported by Rahman et al. (2008).
This study showed that the overall proportion of APEC serotypes to be higher in sick chickens $(52.5 \%)$ compared to healthy chickens (32.3\%), which supports the findings of the previous work from other researchers in this field. Previous study by RodriguezSiek et al. (2005) reported that $70.5 \%$ of $E$. coli were recovered from sick chickens and $57.5 \%$ from faeces of apparently healthy chickens, and recently, Schouler et al. (2012) found that the proportion of the 56.5\% APEC as being from sick chickens and $22.5 \%$ from the faeces of healthy chickens. Mamza et al. (2010) found APEC in only $15 \%$ of sick chickens and $8.8 \%$ of healthy chickens in Nigeria. This rather contradictory result may be due to the geographical location of the study and management strategies of poultry farms (Shankar et al., 2010; Chen et al., 2012). The present findings, though preliminary, suggest that pathogenic E. coli are variably distributed in sick and healthy chickens, but more prevalent in sick chickens than in healthy chickens, suggesting involvement of pathogenic/virulence factors propagating the systemic spread from the gut. Further research should be done to investigate the APEC for virulence factors to identify virulence genes.

The occurrence of antimicrobial resistance among poultry E. coli isolates has been shown to range from 47 to $100 \%$ (Salahi and Bonad, 2006; Jianguo et al., 2012) and multiple resistances ranged from $19.1 \%$ to 72\% (Shtylla et al., 2009; Aggad et al., 2010; Ferdous et al., 2012) in their specific region. The present findings showed high rates of antimicrobial resistant strains to Sulphamethoxazole (98.3\%), Cotrimoxazole (97.7\%) and Ampicillin (42.6\%) in both layers and broilers, which support the findings of the aforementioned studies. Multiple resistances to two or more antibiotics, ranged from 0.6 to $43.2 \%$ in the present study. These findings are lower compared with 
the findings by Ferdous et al. (2012) who found the high multiple resistances of $72 \%$. The observed antimicrobial resistance and multiple resistances may be explained by extensive and inappropriate use of some drugs (Cotrimoxazole 35.1\%, 13/37) and Sulphamethoxazole $(21.6 \%, 8 / 37)$ as mentioned by poultry famers in the study area, as it has been reported elsewhere (Saleha et al., 2009). The prudent use of antimicrobial agents in animals is an essential measure to avoid the occurrence of antimicrobial resistance.

In this study, multiple resistance of $E$. coli to a combination of 4 antibiotics, namely, AmpicillinCotrimoxazole-Sulphamethoxazole-Chloramphenicol among broiler isolates (11.2\%) was significantly higher than layers $(3.4 \%)$ isolates. Similar findings of high levels of multiple resistant isolates in broilers than in layers have been reported elsewhere (Nehme et al., 2005; Wasyl et al., 2013). These data must be interpreted with caution because presumably broilers are exposed to more antibiotics than the layers due to the short span of production. Whether antimicrobials are used in broilers than in layers in the study area remains to be investigated. On the other hand, layers are kept for a long period of time for egg production, especially at an adult age and presumably they experience resistance to pathogenic agents, making them less susceptible to bacterial invasion, thus less need for antibacterial therapy (Nehme et al., 2005).

High frequency of intermediate resistance was observed to Tetracycline (21\%) similar to the findings of Majalija et al. (2010) in Uganda. This finding may be explained that resistance to this drug developed in $E$. coli strains probably due to its frequent use. The use of this drug was observed during sample and data collection as the majority of the poultry keepers $(56.8 \%, 21 / 37)$ mentioned Tetracycline as one of the commonly used drugs. The findings of antimicrobial resistance patterns may be a reflection of the use of those drugs in the study area. These mean that an antibiotic resistant E. coli strain has emerged to a number of antimicrobial agents commonly used by poultry keepers at farm level. Recent study by Katakweba et al. (2012) in Tanzania associated antimicrobial resistance with the misuse of antibiotics in livestock and this may partly be due to lack of knowledge of most of the users, in particular livestock keepers. These findings indicate the risk of antibiotic usage and emphasizes the need for monitoring their indiscriminate use at farm level.

On the contrary, the present findings showed higher rate of antimicrobial sensitivity of $E$. coli to Gentamycin (97.2\%) followed by Streptomycin (85.8\%), Kanamycin (83.6\%), Chloramphenicol $(69.9 \%)$ and Tetracycline $(64.8 \%)$ in both layers and broilers. These findings are contrary to the findings of previous studies (Li et al., 2008; Zakeri and Kashefi,
2012) which recorded high rates of antibiotic resistance to similar drugs ranging from $36.2 \%$ to $93 \%$ in chickens in their specific area. The differences from these other studies may partly explain why these antibiotics are effective in the study area, while they were not effective in the previous study, probably due to extensive use of these antimicrobial agents.

\section{Conclusions and recommendations}

This work has given an account of avian pathogenic E. coli serotypes and provided information on antimicrobial resistance of avian E. coli isolated from healthy and diseased chickens. Despite the limitation of using a small number of serotypes, the study demonstrated the existence of avian pathogenic $E$. coli serotypes O1, O78 and O26 in Tanzania. The study has also shown that there is a high level of resistance to commonly used antimicrobials in the study area, which provides additional evidence to the selection for antimicrobial resistant strains caused by the use of antimicrobials in poultry. Broadly speaking, the present study calls for a wide scale serotyping study, antimicrobial susceptibility studies to clinical samples, serotyping using a wide range of antisera and DNA typing to get more insights about prevailing strains of avian pathogenic $E$. coli and their clinical significance in Tanzania. These efforts should be coupled with a solid disease prevention and control programme, including vaccination, good management and adequate biosecurity measures at the flock level to control diseases in poultry farms.

\section{Acknowledgements}

The authors are grateful to the Ministry of Livestock and Fisheries Development and Tanzania Veterinary Laboratory Agency for the financial support which enabled to carry out this research. However, special thanks go to TVLA staff for their moral assistance in laboratory work and Livestock field officers from Arusha City Veterinary Office, for their assistance during sample and data collection.

\section{References}

Aggad H, Ammar H, Kihal M (2010) Antimicrobial resistance of $E$. coli isolated from chickens with colibacillosis. Glob Vet 4(3): 303-306.

Allan BJ, Hurk JV, Potter AA (1993) Characterization of E. coli isolated from cases of avian colibacillosis. Canad J Vet Res 57: 146-151.

Blanco JE, Blanco M, Mora A, Blanco J (1997) Prevalence of bacterial resistance to quinolones and other antimicrobials among avian E. coli strains isolated from septicemic and healthy chickens in Spain. J Clin Microbiol 35: 2184-2185. 
Centers for Disease Control and Prevention (2012) Statistical software for public health professionals. Retrieved April 20, 2012 from [http://wwwn.cdc.gov/epiinfo/7/index.htm]

Cheesbrough M (2000) District Laboratory Practice for Tropical Countries. Cambridge University Press, UK. P:179

Chen X, Yin J, Huan H, Gao S, Jiao X, Liu X (2012) Serogroups, pathogenicity and virulence-associated genes of avian E. coli isolates collected in China. Afr J Microbiol Res 6(5): 1001-1007.

Clinical and Laboratory Standards Institute (2011) Performance Standards for Antimicrobial Susceptibility Testing, Twenty First Informational Supplement CLSI document Paper No. 21, Clinical and Laboratory Standards Institute. Wayne, USA. $\mathrm{P}: 165$.

Dheilly A, Devendec L, Mourand G, Bouder A, Jouy E, Kempf I (2012) Resistance gene transfer during treatments for experimental avian colibacillosis. Antimicrob Agents Chemother 56(1): 189-196.

D'Incau M, Pennelli D, Lavazza A, Tagliabue (2006) Serotypes of $E$. coli isolated from avian species in Lombardia and Emilia Romagna North Italy. Ital J Anim Sci 5: 298-301.

Fairbrother JM, Nadeau E (2006) Escherichia coli: Onfarm contamination of animals. Review Scientific Tech Office Intern Epizootic 25(2): 555-569.

Ferdous T, Hossain KMM, Kabir SLM, Amina MM (2012) Characterization in pluck shops at Sreepur of Gazipur district in Bangladesh of E. coli isolates obtained from washing and rinsed water of Broilers. Sci J Microbiol 1(5): 126-132.

Geidam YA, Ambali AG, Onyeyili PA (2012) Detection and antibiotic sensitivity pattern of avian pathogenic E. coli strains among rural chickens in the Arid region of North-Eastern Nigeria. Vet World 5(6): 325-329.

Gross W (1994) Diseases Due to E. coli in Poultry. In: Esherichia coli in domestic animals and humans. CAB International, Wallingford, UK. pp: 237-259.

Ivanov ID (2007) Diseases of Poultry, A colour Atlas, $1^{\text {st }}$ Edition. Ceva Sante Animale, Bulgaria. P:22.

Jianguo Y, Weihua C, Huafu W, Wei Z (2012) Antimicrobial susceptibility and virulence factors of $E$. coli isolates obtained from faeces samples of chickens in east China. Afr J Micrbiol Res 6(7): $1591-1596$.

Johnson LC, Bilgili SF, Hoerr FJ, McMurtrey BL, Norton RA (2001) The influence of E. coli strains from different sources and the age of broiler chickens on the development of cellulitis. Avian Pathol 30: 475-479.

Katakweba AAS, Mtambo MMA, Olsen JE, Muhairwa AP (2012) Awareness of human health risks associated with the use of antibiotics among livestock keepers and factors that contribute to selection of antibiotic resistance bacteria within livestock in Tanzania. Liv Res Rur Dev 24:170.

Khoo L, Hasnah Y, Rosnah Y, Saiful N, Maswati M, Ramlan M (2010) The prevalence of avian pathogenic E. coli in Peninsular Malaysia. Malay J Vet Res 1: 27-31.

Kok T, Worswick D, Gowans V (1996) Serological techniques for microbial and viral infections, In: Mackie and McCartney Practical Medical Microbiology $14^{\text {th }}$ Edition. Churchill Livingstone, New York, USA. pp: 179-384.

Li S, Yi-baol N, Xu-yingl Z, Qing-chunl S, Zhang Guang-chuan Z, Shu-mao L, Hao-tingl W, Huil Z, Guangl G, Zhong-wul F (2008) Comparative research on serogroups distribution and antimicrobial resistance of $E$. coli isolates from poultry in different areas of China. Agric Sci Chin 7(3): 381-386.

Longman AW (1999) Microbiology: A Laboratory Manual, $5^{\text {th }}$ Edition. Benjamin Cummings Science Publishing, Menlo Park, Califonia, P:471.

Majalija S, Francis O, Sarah WG, Luboma M, Vudriko P, Nakamya (2010) Antibiotics susceptibility profiles of faecal $E$. coli isolates from deep litter broiler chickens in Northern and Central Uganda. Vet Res 3(40): 75-80.

Mamza SA, Egwu GO, Mshelia GD (2010) Antibiotic susceptibility patterns of beta-lactamase producing Escherichia coli and Staphylococcus aurius isolated from chickens inMaidugur (Arid zone) Nigeria. Vet Arhiv 80(2): 283-297.

Merchant IA, Parker RA (1967) Veterinary Bacteriology and Virology, $7^{\text {th }}$ Edition. Central Board of Secondary Education Publishers, New Delhi, India. P:750.

Nehme PA, Barbour EK, Sagherian VK, Shadi K, Hamadeh SK, Zurayk RK (2005) Tracheal bacterial flora and drug susceptibility in chicken reared under different systems. Int J Appl Res Vet Med 3(4): 372-377.

Pfeiffer DV (1999) Veterinary epidemiology An introduction, Epicenter, New Zealand. P:345.

Rahman M, Rahman BM, Rahman B (2008) Antibiogram and plasmid profile analysis of isolated E. coli from broilers and layers. Res J Microbiol 3: 82-90.

Rodriguez-Siek KE, Giddings CW, Doetkott C, Timothy J, Johnso TJ, Lisa K, Nolan LK (2005) Characterizing the APEC Pathotype. Vet Res 36: 241-256.

Saleha AA, Myaing TT, Ganapathy KK, Zulkifli I, Raha R, Arifah K (2009) Possible effect of antibiotic-supplemented feed and environment on the occurrence of multiple antibiotic resistant $E$. coli in chickens. Int J Poult Sci 8(1): 28-31. 
Salehi TZ, Bonab F (2006) Antibiotics susceptibility pattern of $E$. coli strains isolated from chickens with colisepticemia in Tabriz Province, Iran. Int $\mathbf{J}$ Poult Sci 5(7): 677-684.

Schouler C, Schaeffer B, Brée A, Mora A, Dahbi G, Biet F, Oswald E, Mainil J, Blanco J, MoulinSchouleur M (2012) Diagnostic strategy for identifying avian pathogenic $E$. coli based on four patterns of virulence genes. J Clin Micrbiol 50: 1673-1678.

Shankar TV, Sharma A, Grover Y (2010) Studies on different virulence factors of avian pathogenic $E$. coli. Haryana Vet 49: 45-47.

Shtylla T, Circella E, Madio A, Di Paola G, Çabeli P, Kumbe I, Kika A, Camarda A (2009). Multiple antimicrobial resistance among avian $E$. coli strains in Albania. Ital J Anim Sci 8: 771-774.
Stephen JC, Ronald JH, Yvette SM, José HO, Ivonne DR, Robert LS, Susan ES, Carol AS (2002) Manual of Antimicrobial Susceptibility Testing. University of Washington Seattle, Washington, DC, p: 236.

Wasyl D, Hoszowski A, Zajac M, Szulowski K (2013) Antimicrobial resistance in commensal E. coli isolated from animals at slaughter. Frontiers Microbiol 4(221): 1-12.

Yousseff FM, Ahmed MA, Mansour DH (2008) Clinical, pathological and bacteriological investigations on air sacculitis in chickens in Ismailia Province (Egypt). J Agric Vet Sci 1(2): 71-79.

Zakeri A, Kashefi P (2012) Antimicrobial susceptibilities of avian E. coli isolates in Tabriz, Iran. Afr J Biotech 11(19): 4467-4470. 